\title{
Mutant Exon1 Huntingtin Aggregation is Regulated by T3 Phosphorylation-Induced Structural Changes and Crosstalk between T3 Phosphorylation and Acetylation at K6
}

\section{Authors}

Anass Chiki, Sean M. DeGuire,Francesco S. Ruggeri, Domenico Sanfelice, Annalisa Ansaloni, Dr. ZheMing Wang, Urszula Cendrowska, Dr. Ritwik Burai, Sophie Vieweg, Prof. Annalisa Pastore, Prof. Giovanni Dietler, Prof. Hilal A. Lashuel

\section{Abstract}

Herein, we used protein semisynthesis to investigate, for the first time, the effect of lysine acetylation and phosphorylation, as well as the crosstalk between these modifications on the structure and aggregation of mutant huntingtin exon1 (Httex1). Our results demonstrate that phosphorylation at T3 stabilizes the $\alpha$-helical conformation of the N-terminal 17 amino acids (Nt17) and significantly inhibits the aggregation of mutant $\mathrm{Httex1}$. Acetylation of single lysine residues, K6, K9 or K15, had no effect on Httex1 aggregation. Interestingly, acetylation at K6, but not at $\mathrm{K} 9$ or $\mathrm{K} 15$, reversed the inhibitory effect of T3 phosphorylation. Together, our results provide novel insight into the role of Nt17 posttranslational modifications in regulating the structure and aggregation of Httex1 and suggest that its aggregation and possibly its function(s) are controlled by regulatory mechanisms involving crosstalk between different PTMs.

\section{Main text}

Several lines of evidence suggest that the first $\mathrm{N}$-terminal 17 amino acids of the Huntingtin protein (Htt) play important roles in modulating $\mathrm{Htt}$ structure, oligomerization, fibrils formation, membrane binding, subcellular localization and interactions with other proteins.[1] This, combined with the fact that this short $\mathrm{N}$-terminal sequence harbors several residues that are subjected to diverse posttranslational modifications (PTMs), including phosphorylation, acetylation, ubiquitination and sumoylation (Scheme S1-A in the Supporting Information), suggests that PTMs may serve as reversible molecular switches for regulating $\mathrm{Htt}$ structure, interactome, cellular properties and toxicity.[2] However, the lack of knowledge about the enzymes involved in regulating these modifications has made it difficult to decipher their role in regulating the function(s) of $\mathrm{Htt}$ in health and disease.

To address these limitations and enable deciphering of the Nt17 PTM code, our group has pursued the development of semisynthetic strategies that permit site-specific introduction of single or multiple PTMs within Nt17 of Huntingtin exon 1 (Httex1). Recently, we reported a semisynthetic strategy that permits site-specific phosphorylation at T3.[3] However, this strategy suffered from several limitations and was only suitable for introducing PTMs in the first $\mathrm{N}$-terminal 9 residues of wild-type Httex1 ( $\leq 23$ glutamine residues) (Scheme S2).[3] To allow investigations of all Nt17 PTMs, we developed and optimized two new modular semisynthetic protocols that permit the generation of wild-type and for the first time mutant Httex1 (mHttex1) proteins containing single or multiple PTMs (Scheme S3). The first strategy (Scheme S3-A) is only suitable for introducing PTMs in the N-terminal 9 residues of $\mathrm{Httex1}$, whereas the second strategy allows the investigation of all the PTMs within the Nt17 domain. Additionally, the optimized conditions enabled the semisynthesis of mutant Httex1 (full details about these semisynthetic strategies are given in the Supporting Information). This optimized strategy enabled us, for the first time, to generate milligram quantities of $\mathrm{mHttex1}$ (43Q) phosphorylated at T3 (pT3) or acetylated at K6, K9 or K15 (AcK6, AcK9, AcK15), as well as Httex1 containing multiple combination of both PTMs (pT3/AcK6, pT3/AcK9, pT3/AcK15) to probe the potential of cross-talk between these modifications. 
Threonine 3 (T3) has been identified as one of the most common phosphorylation sites in Htt from ST14A and HeLa cells and was detected at lower levels in the post-mortem brain regions most affected by HD in a mouse model of HD (CAG140).[4, 5] Interestingly, the level of T3 phosphorylation was inversely correlated with polyQ repeat length, suggesting that phosphorylation at $\mathrm{T} 3$ is decreased in pathological conditions and that restoring the physiological level of T3 phosphorylation might ameliorate HD pathology.[4] Testing this hypothesis has not been possible because the enzymes involved in regulating T3 phosphorylation remain elusive and the extent to which phosphomimetic mutations can reproduce the effect of phosphorylation remains unknown.

To address this knowledge gap, we sought to 1) determine the effect of T3 phosphorylation on mHttex1 aggregation and 2) to determine whether the phosphomimetic mutation (T3D/E), which is commonly used to assess T3 phosphorylation in cellular and animal models of HD, reproduces the effects of authentic T3 phosphorylation on the structure and aggregation of mutant Httex1. To achieve these goals, we prepared pT3 and T3D mHttex1-43Q using the new semisynthetic strategy (Figure S6). Sedimentation analysis of the unmodified mHttex1-43Q showed that circa $75 \%$ of the protein aggregated within $12 \mathrm{~h}$ at $37^{\circ} \mathrm{C}$ and that nearly complete depletion of the soluble protein fraction occurred between 24 and $36 \mathrm{~h}$ (Figure $1 \mathrm{~A}$ ). This was accompanied by a shift in the circular dichroism (CD) spectrum that is indicative of a change in secondary structure from a predominantly disordered structure $(\lambda \min 205 \mathrm{~nm}$ ) to a $\beta$-sheet-rich structure $(\lambda \min 215 \mathrm{~nm}$, Figure $1 \mathrm{~B})$, consistent with the observation of fibrillar aggregates at $48 \mathrm{~h}$ by transmission electron microscopy (TEM) (Figure $\mathrm{S} 7 \mathrm{and}$ S8). In contrast, $89 \%$ and $>60 \%$ mHttex1-pT3 remained soluble after 12 and $48 \mathrm{~h}$ of incubation, respectively (Figure $1 \mathrm{~A}$ ). No major change in the secondary structure of Httex1-pT3 was observed by $\mathrm{CD}$ even after $48 \mathrm{~h}$ (Figure $1 \mathrm{~B}$ ). TEM analysis showed mainly oligomers and short fibrillar structures with shorter average lengths than those formed by unmodified mHttex1 (Figure S7 and S8). Similar inhibitory effects were observed at higher protein concentrations. Fibrils formation by mHttex1-pT3 was only observed at concentrations of $\geq 30 \mu \mathrm{m}$ (Figure S9). Interestingly, the strong $\beta$-sheet and aggregation inhibitory effect induced by phosphorylation at T3 were not fully reproduced by the phosphomimetic mutation (T3D) (Figure 1). The fibrils formed by mHttex1-T3D were of intermediate size (Figure S7) compared to those formed by pT3 and unmodified mHttex1 (Figure S7 and S8). Detailed characterization of the morphology and dimensions of the aggregates formed by PT3 and T3D by high-resolution atomic force microscopy (AFM) are presented in Figure S10. After $48 \mathrm{~h}$ of incubation, unmodified $\mathrm{mHttex} 1$ formed mature fibrillar species (Figure $1 \mathrm{C}$ ) with an average length of $189 \pm 103 \mathrm{~nm}$ and height of $6 \pm 1 \mathrm{~nm}$ (Figure $1 \mathrm{D}$ ). The $\mathrm{mHttex1-T3D}$ exhibited shorter fibrils (106 \pm 49

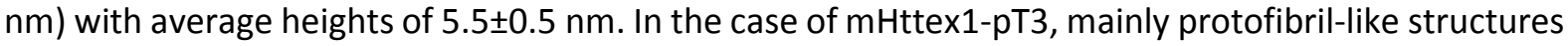
with average lengths and heights of $71 \pm 30 \mathrm{~nm}$ and $4.6 \pm 0.5 \mathrm{~nm}$ were observed (Figure $1 \mathrm{D}$ ). These observations suggest that the phosphomimetic mutation reproduces only partially the effect of phosphorylation at T3.

To gain insight into the mechanism by which this modification inhibits mutant Httex1 aggregation, we investigated the effects of phosphorylation and phosphomimetics at T3 on the structure of Nt17 peptides. Htt1-19 showed a CD spectrum indicative of a mainly random coil structure mixed with partially $\alpha$-helical structures consistent with previous $C D$ and atomistic simulation studies (Figure 2 A). $[6,7]$ In contrast, the CD spectrum of Htt1-19-pT3 showed a double minimum at 208 and $222 \mathrm{~nm}$, indicative of a predominantly $\alpha$-helical structure (Figure $2 \mathrm{~A}$ ). To the best of our knowledge, this is the most striking stabilization of Nt17 helical conformation without the use of fluorinated solvents (e.g. TFE). The pT3-induced $\alpha$-helical structure is $\mathrm{pH}$-sensitive. $\mathrm{N}$-terminal acetylation increases the pT3induced helicity, but the overall helicity follows the same linear decrease with decreasing $\mathrm{pH}$ in the presence or absence of acetylation (Figure 2 B). The HttAc2-19 T3E and T3D peptides exhibited CD 
spectra similar to that of the unmodified HttAc2-19 peptide (Figure $2 \mathrm{~A}$ ), confirming again that these mutations do not reproduce the effects of phosphorylation at T3 on the structural properties of Nt17.

Next, we conducted nuclear magnetic resonance (NMR) studies of the conformation of Htt1-19 and Htt1-19pT3 (Table S2) in aqueous buffer and in 2,2,2-trifluoroethanol (TFE) (33\%) and compared their chemical shifts and the NOESY patterns.[8] The spectra of Htt1-19 and Htt1-19pT3 have similar features in aqueous buffer and TFE with similar chemical shifts for residues 6-19. However, the amide proton of T3 appears significantly shifted in Htt1-19 pT3, which is highly shifted downfield at approximatively $9.9 \mathrm{ppm}(>1.5 \mathrm{ppm})$ compared to the unmodified peptide (Figure $2 \mathrm{C}, \mathrm{D}$, left). Phosphorylated peptides do not typically present such a chemical shift variation. Even more notable is the NOE pattern. In buffer (Figure 2 C,D, right), the spectrum of Htt1-19 has clear features of a nascent helix (HN-HN connectivity) that can be stabilized by fluoroalcohols (TFE) demonstrating a propensity to assume a stable $\alpha$-helical conformation. The NOESY spectra of Htt1-19 in TFE (Figure $2 \mathrm{E}$ ) show uninterrupted sequential $\mathrm{HN}-\mathrm{HN}$ connectivities and other NOE patterns expected for helical structures ( $\mathrm{HNi}-\mathrm{HNi}+1, \mathrm{H \alpha i}-\mathrm{HNi}+4$ and $\mathrm{H \alpha i}-\mathrm{HNi}+4)$ in the tract 5-19. Residues $\mathrm{M} 1, \mathrm{~A} 2$ and $\mathrm{T} 3$ are not identifiable indicating a high flexibility of the N-terminus where the helix frays. In the NOESY of Htt119pT3, the resonance of the T3 amide of Htt1-19pT3 forms unmistakable sequential HN-HN connectivities with the previous and following amide groups, indicating that the phosphorylated T3 residue is strongly rigidified compared to the unmodified residue extending $\alpha$-helix structure to $A 2$.

Although acetylation of lysine residues within $\mathrm{Nt17}$ has been proposed to play a role in regulating its association with membranes,[9] only $\mathrm{K9}$ was reported to be acetylated in HEK cells[10] and was proposed to play an important role in Htt clearance.[11] Chaibva et al. reported that acetylation of Httex1 retards fibrils formation in vitro.[9] However, it is noteworthy that their findings were based on nonselective chemical acetylation of all lysine residues in a model peptide of Httex1 lacking the last 39 C-terminal residues and containing two non-native lysines residues which were also partially acetylated. To investigate the effect of lysine acetylation on Httex1 aggregation, we produced both WT (23Q) and mutant (43Q) Httex1 acetylated at single lysine residues (AcK6, AcK9, and AcK15). Figure 3 demonstrates that acetylation at single lysine residues does not significantly influence the aggregation of WT or mutant Httex1 (Figure 3, Figure S8 and S11), except for acetylation at K6 that appeared to slightly delay mHttex1 aggregation. Although we observed a trend of a slightly accelerated aggregation of $\mathrm{Httex} 1$ acetylated at $\mathrm{K} 9$ and $\mathrm{K} 15$, the differences in aggregation kinetics compared to the unmodified Httex1 were not statistically significant. In all cases, the loss of soluble protein was accompanied by a shift in the secondary structure from random coil to $\beta$-sheet structure (Figure $3 \mathrm{~B}$ ) and by the formation of fibrillar aggregates of comparable morphology and length (Figure $3 \mathrm{C}$, Figure S8). These findings suggest that lysine acetylation at K6, K9 or K15 alone does not play a critical role in the regulation of $\mathrm{Httex1}$ aggregation in vitro.

Given the close proximity of lysine residues K6, K9 and K15 to neighboring phosphorylation sites (T3, S13 and S16), we examined the potential crosstalk between lysine acetylation and phosphorylation within Nt17. We focused initially on phosphorylation at T3 and acetylation at K6 because of the close proximity of the two residues, also due to the role of $\mathrm{K} 6$ in stabilizing pT3-induced Nt17 helix formation and the striking inhibitory effect of pT3 on mHttex 1 aggregation. Sedimentation analysis showed that mHttex1-pT3/AcK6 exhibited similar aggregation profile as unmodified mHttex1 and mHttex1-AcK6 (Figure $3 \mathrm{~A}$ ), whereas mHttex1-pT3/AcK9 and mHttex1- pT3/AcK15 Q18A, exhibited similar

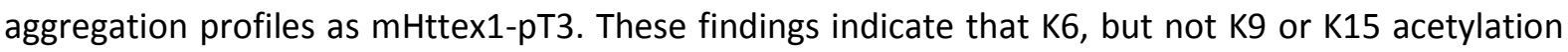
reverses the inhibitory effect of T3 phosphorylation on mutant Httex1 aggregation. Consistent with this hypothesis, only mHttex1-pT3/AcK6 converted to $\beta$-sheet-rich fibrillar structures after $48 \mathrm{~h}$ of incubation, whereas mutant mHttex1-pT3, mHttex1-pT3/AcK9 or mHttex1-pT3/AcK15 exhibited CD 
spectra consistent with a predominantly disordered conformation (Figure $3 \mathrm{~B}, \mathrm{C}$ and S12). mHttex1pT3/AcK6 and mHttex1-AcK6 formed predominantly long mature fibrils of similar size (height $>6 \mathrm{~nm}$, length 50-350 nm), whereas mHttex1-pT3 formed predominantly short protofibrillar species (height $<5 \mathrm{~nm}$, length $50-150 \mathrm{~nm}$ ) (Figure S13). Similar observations were made at higher protein concentrations (30-45 $\mu \mathrm{m}$, Figure S14). To determine if the effect of acetylation at $K 6$ on the aggregation inhibitory effects of phosphorylation at T3, we investigated by NMR the role of K6 in stabilizing/destabilizing the $\mathrm{N}$-terminal helical conformation induced by pT3. We compared the behavior of the N-terminally protected phosphorylated and non-phosphorylated AcHtt2-17AcK6 peptides, where $\mathrm{K} 6$ was neutralized with acetylation, to that of AcHtt2-17. As it has become clear to us that native $\mathrm{Htt}$ is $\mathrm{N}$-terminally acetylated, all the peptides used to investigate the interplay between T3 phosphorylation and $\mathrm{K} 6$ acetylation were $\mathrm{N}$-terminally acetylated. As shown in Figure $2 \mathrm{~A}, \mathrm{~N}-$ terminal acetylation does not significantly alter the conformational properties of Nt17. Both AcHtt217pT3 and AcHtt2-17pT3/AcK6 present the resonance of pT3 down-field shifted as compared to AcHtt2-17 and AcHtt2-17AcK6 (Figure S15). However, the NOESY pattern of AcHtt2-17pT3 and AcHtt217pT3/AcK6 are similar, indicating that acetylation at $\mathrm{K} 6$ does not significantly alter the Nt17 helical conformation (Figure S15). Our NMR results are confirmed by the CD analysis of the same peptides, which showed that pT3 and pT3/AcK6 Httex1 exhibit similar helical structure and contents (Figure S16). These findings are consistent with emerging evidence that the role of the Nt17 in regulating $\mathrm{Httex} 1$ aggregation is determined by factors that influence not only the conformation of this peptide but also its interactions with itself and the polyQ domain. $[6,12,13]$ The existing data do not support a direct and simple relationship between Nt17 helicity and Httex1 aggregation, suggesting that Httex1 aggregation can proceed through distinct amyloidogenic conformations. Therefore, more studies are needed to dissect the different mechanisms by which PTMs or crosstalk between PTMs influence the intra- and intermolecular interactions of $\mathrm{Httex1}$, the amyloidogenic conformation of the polyQ domain and Httex1 aggregation.

We have described herein the first semisynthesis of mutant Httex1 (43Q). Our improved method allows the production of milligram quantities of mutant Httex1 and enables the homogeneous Httex1 preparations with site-specifically modifications at single or multiple residues. This enabled us for the first time, to perform detailed studies comparing the effects of phosphorylation at $\mathrm{T} 3$ and acetylation at K6, K9 or K15 and assessing the crosstalk between the two modifications (pT3/AcK6, pT3/AcK9 and pT3/AcK15) on the Httex1 aggregation. Our results demonstrated that acetylation of single lysine residues within the $\mathrm{N}$-terminal 17 amino acids of Httex1 did not significantly modify the aggregation kinetics or structural properties of wild-type or mutant Httex1. In contrast, phosphorylation at T3 resulted in dramatic stabilization of $\mathrm{Nt17}$ helical conformation and inhibition of mutant Httex1 aggregation. In contrast the The phosphomimetic T3D mutation had no effect on Nt17 conformation and resulted in only intermediate inhibition of Httex1, demonstrating that this mutation does not reproduce the structural and aggregation properties of authentic phosphorylation. Finally, we showed that K6, but not $\mathrm{K} 9$ or $\mathrm{K} 15$ acetylation reverses the inhibitory effect of T3 phosphorylation on Httex1 aggregation. Our results suggest that phosphorylation/dephosphorylation of $\mathrm{T} 3$ and/or the crosstalk between T3 phosphorylation and acetylation represent PTM-dependent molecular switches for regulating the structure of $\mathrm{Nt} 17$ and possibly the aggregation of $\mathrm{Htt}$ and $\mathrm{N}$-terminal Htt fragments in vivo.

Acknowledgements 
This work was supported by the CHDI foundation (A_7627) and the Swiss National Science Foundation (31003A-146680). We thank Dr. Mahmood Haj-Yahya, Dr. John Warner, Dr. Elizabeth Doherty, Dr. Celia Dominguez and Dr. Andrea Caricasole for helpful discussions on the manuscript, and IRBM for generating the Nt17 peptides used in this study. We are grateful to $\mathrm{Dr}$ G. Kelly and $\mathrm{Dr} \mathrm{T}$. Frenkiel at the MRC Biomedical NMR Centre (The Francis Crick Institute) for providing access to the NMR facilities and help in acquiring spectra.

Conflict of interest

The authors declare no conflict of interest.

References

1 J. R. Arndt, M. Chaibva, J. Legleiter, Biomol. Concepts 2015, 6, 33-46.

2 D. E. Ehrnhoefer, L. Sutton, M. R. Hayden, Neuroscientist 2011, 17, 475-492.

3 A. Ansaloni, Z. M. Wang, J. S. Jeong, F. S. Ruggeri, G. Dietler, H. A. Lashuel, Angew. Chem. Int. Ed. 2014, 53, 1928-1933;

4 C. T. Aiken, J. S. Steffan, C. M. Guerrero, H. Khashwji, T. Lukacsovich, D. Simmons, J. M. Purcell, K. Menhaji, Y. Z. Zhu, K. Green, F. Laferla, L. Huang, L. M. Thompson, J. L. Marsh, J. Biol. Chem. 2009, 284, 29427-29436.

5 M. B. Bustamante, A. Ansaloni, J. F. Pedersen, L. Azzollini, C. Cariulo, Z. M. Wang, L. Petricca, M. Verani, F. Puglisi, H. Park, H. Lashuel, A. Caricasole, Biochem. Biophys. Res. Commun. 2015, 463, 13171322.

6 T. E. Williamson, A. Vitalis, S. L. Crick, R. V. Pappu, J. Mol. Biol. 2010, 396, 1295-1309.

7 R. S. Atwal, C. R. Desmond, N. Caron, T. Maiuri, J. R. Xia, S. Sipione, R. Truant, Nat. Chem. Biol. 2011, 7, 453-460.

8 M. Buck, Q. Rev. Biophys. 1998, 31, 297-355.

9 M. Chaibva, S. Jawahery, A. W. Pilkington, J. R. Arndt, O. Sarver, S. Valentine, S. Matysiak, J. Legleiter, Biophys. J. 2016, 111, 349-362.

10 X. Cong, J. M. Held, F. DeGiacomo, A. Bonner, J. M. Chen, B. Schilling, G. A. Czerwieniec, B. W. Gibson, L. M. Ellerby, Mol. Cell. Proteomics 2011, 10, M111 009829.

11 L. M. Thompson, C. T. Aiken, L. S. Kaltenbach, N. Agrawal, K. Illes, A. Khoshnan, M. MartinezVincente, M. Arrasate, J. G. O'Rourke, H. Khashwji, T. Lukacsovich, Y. Z. Zhu, A. L. Lau, A. Massey, M. R. Hayden, S. O. Zeitlin, S. Finkbeiner, K. N. Green, F. M. LaFerla, G. Bates, L. Huang, P. H. Patterson, D. C. Lo, A. M. Cuervo, J. L. Marsh, J. S. Steffan, J. Cell Biol. 2009, 187, 1083-1099.

12 S. L. Crick, K. M. Ruff, K. Garai, C. Frieden, R. V. Pappu, Proc. Natl. Acad. Sci. USA 2013, 110, 2007520080.

13 S. Tam, C. Spiess, W. Auyeung, L. Joachimiak, B. Chen, M. A. Poirier, J. Frydman, Nat. Struct. Mol. Biol. 2009, 16, 1279-1285. 
Figures

A
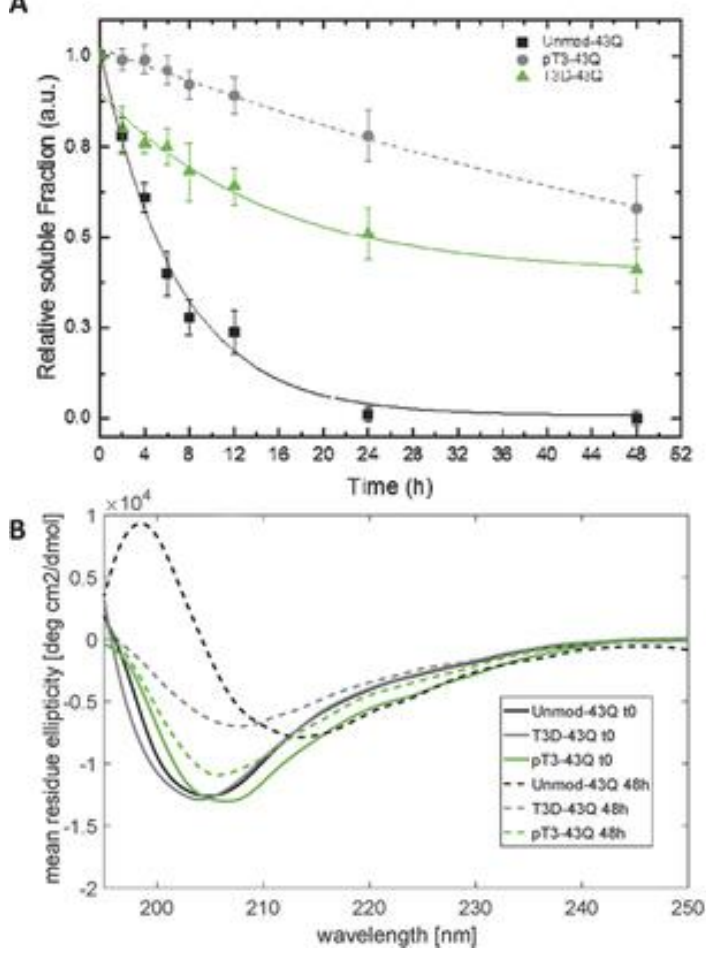

c

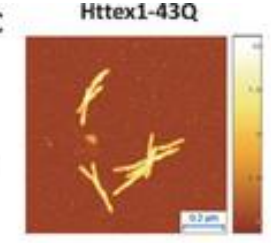

48h

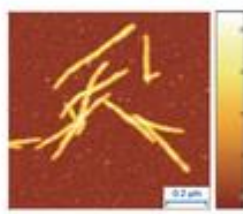

D

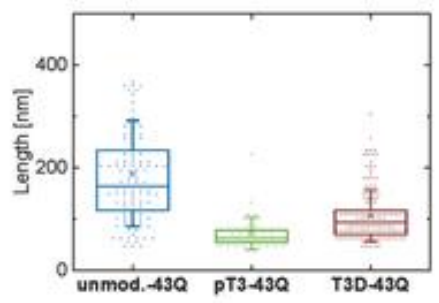

Httex1-43Q pT3
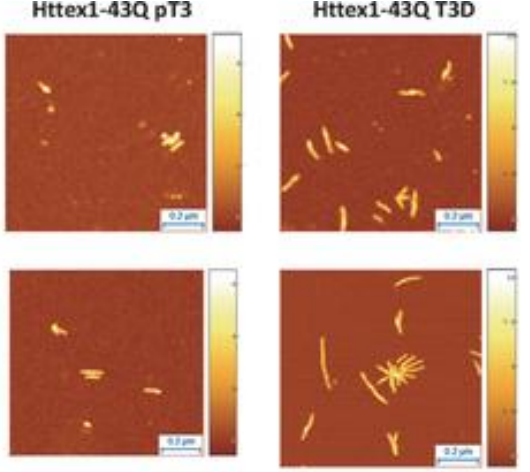

Httex1-43Q T3D

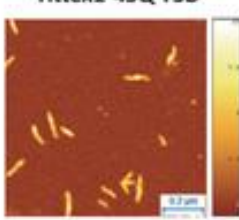

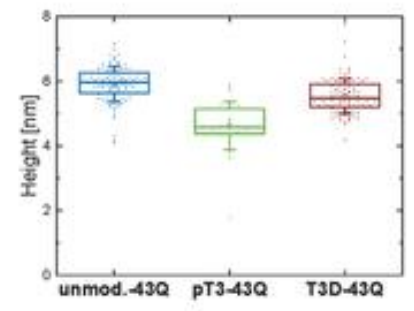

Figure 1. Aggregation of mHttex1-pT3 and T3D compared to unmodified mHttex1 at $3 \mu \mathrm{m}$. A) Sedimentation assay (loss of soluble protein) based on RP-UPLC ( $n=6$, fitted with exponential decay). B) CD analysis of the samples at 0 and $48 \mathrm{~h}$. C) AFM characterization of unmodified mHttex 1 and mHttex1-pT3 and T3D aggregates. The XY scale bars are $200 \mathrm{~nm}$, the $Z$ scales are in $\mathrm{nm}$. D) Height and length quantification of the aggregates formed by the three proteins at $48 \mathrm{~h}$. 

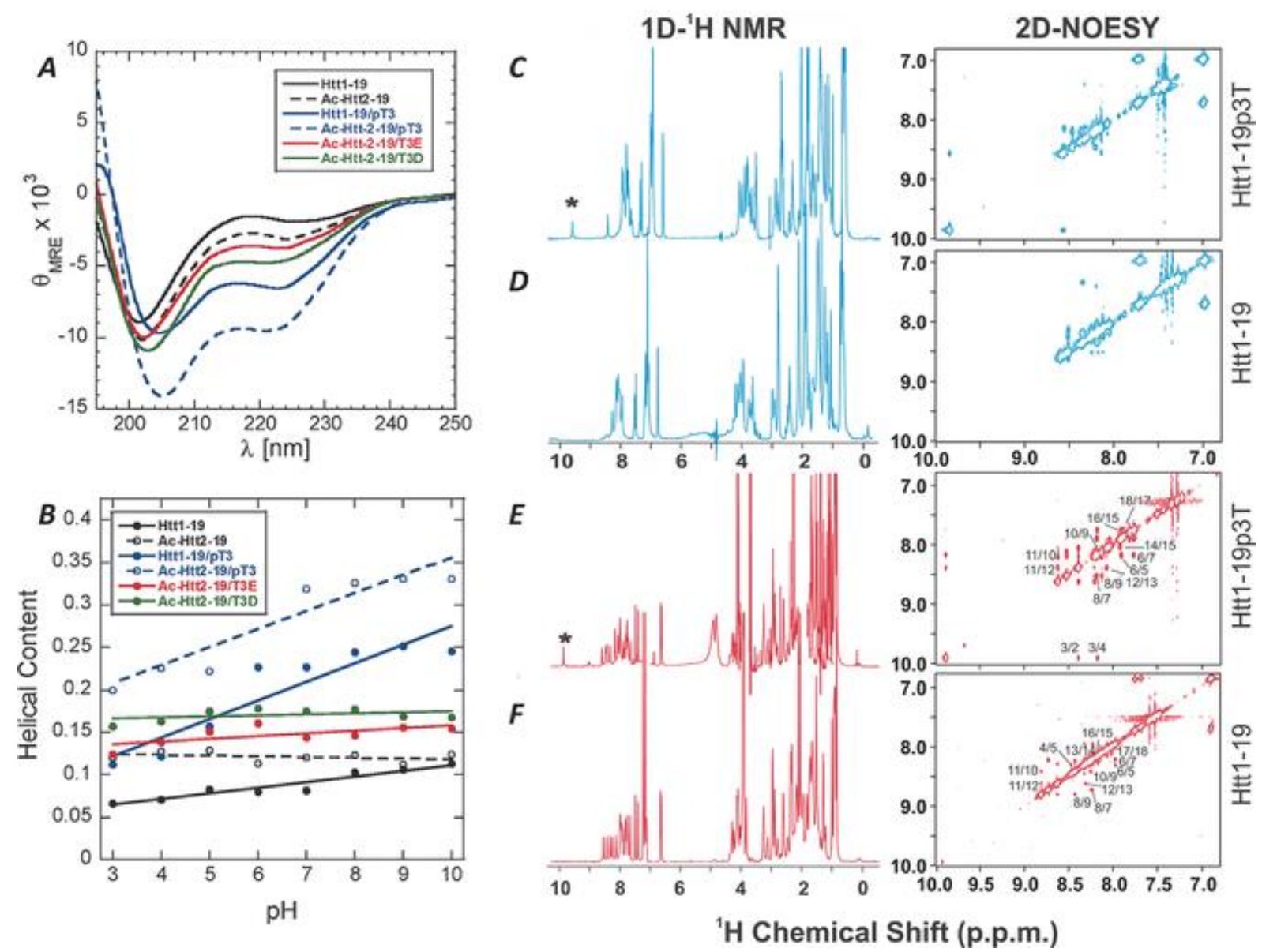

Figure 2. NMR and circular dichroism analysis of the Htt1-19 peptides. A) Far-UV CD spectra of WT and pT3 Htt1-19 peptides compared to WT, pT3, T3E and T3D HttAc2-19 at pH 7.0 in aqueous buffer. B) Helical content of each peptide as a function of pH. C and D) 1D WATERGATE and 2D homonuclear NOESY spectra of Htt1-19 phosphorylated in position 3 and non-phosphorylated, respectively. $\mathrm{E}$ and F) Same as in (C) and (D) in the presence of $33 \%$ TFE: also reported is the assignment of the NH-NH chemical shifts typical of $\alpha$-helical structure, a star highlights the characteristic signals arising upon phosphorylation of T3. 


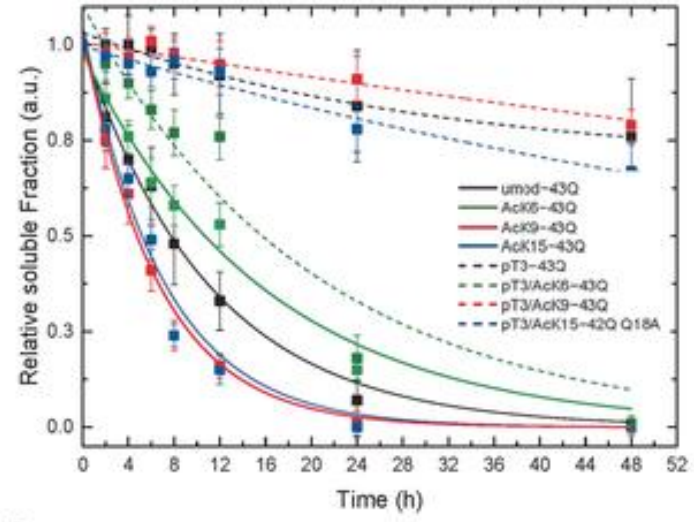

B

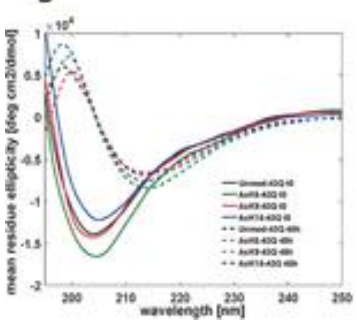

C
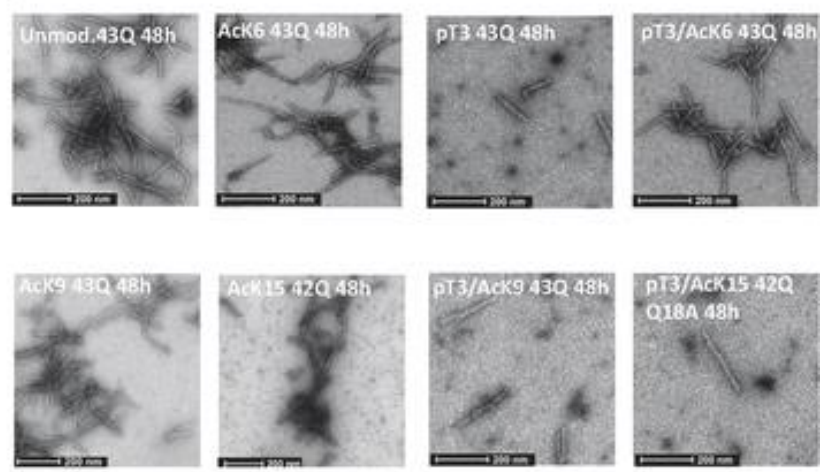

D

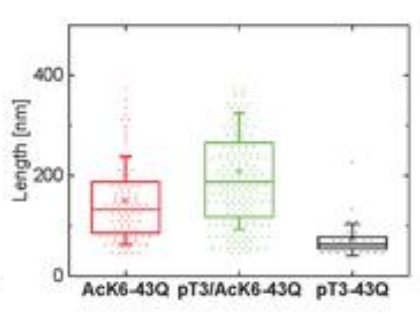

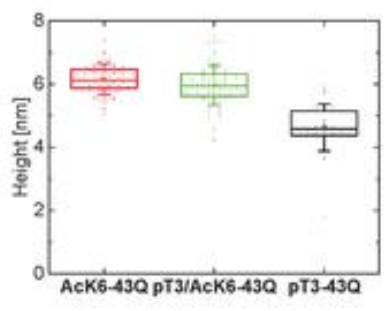

Figure 3. Aggregation of single lysine acetylated mHttex1 and mHttex 1 phosphorylated at $\mathrm{T} 3$ and acetylated at-AcK6, AcK9, or AcK15. A) Sedimentation assay (loss of soluble protein) based on RP-UPLC $(n=6$, fitted with exponential decay). B) CD analysis and C) TEM images of the proteins after $48 \mathrm{~h}$ of aggregation. D) Height and length quantification of the aggregates by AFM at $48 \mathrm{~h}$. 